\title{
COMUNICACIÓN/COMMUNICATION
}

Recibido: 24/11/2011---Revisado: 10/01/2012

Aceptado: 19/04/2012---Publicado: 15/07/2012

\section{LENGUAJE DEPORTIVO Y COMUNICACIÓN SOCIAL: PROTOTIPO COETÁNEO DE MASAS}

Carlos Oliva Marañón: Universidad Rey Juan Carlos (Madrid). España. carlos.oliva.maranon@urjc.es

\section{RESUMEN}

El deporte se concibe como una auténtica revolución copernicana, interpretado como teoría de la comunicación y, por tanto, es parte de la estructura misma de la sociedad actual. El interés por el lenguaje deportivo se fundamenta en el paradigma de que idioma y deporte constituyen dos formas de inteligencia y libertad. Hoy, el reto de las Nuevas Tecnologías, la globalización del deporte y su gran creatividad lingüística configuran la necesidad de controlar y proteger el lenguaje. El objetivo de esta investigación es conocer cuáles son las principales incorrecciones lingüísticas de la información deportiva en la prensa digital. Estas desviaciones de la norma atentan contra la corrección idiomática de la Lengua Española. Por un lado, la investigación revela que la prensa deportiva incluye términos de lenguaje violento, metáforas, galicismos, anglicismos, omisión del artículo y uso incorrecto de las preposiciones. Por otro lado, en el lenguaje deportivo se constata la presencia de términos de ámbitos diversos como la religión, la salud, la épica o la música. Las razones de estas incorrecciones léxicas son diversas: despreocupación por el buen uso del idioma y limitación de espacio en las noticias. Todo ello, afecta al ámbito de las Humanidades $y$, por consiguiente, a la sociedad.

PALABRAS CLAVE: Lenguaje deportivo - Prensa deportiva - Lengua Española Extranjerismos - Nuevas Tecnologías

\footnotetext{
${ }^{1}$ Carlos Oliva Marañón: Profesor de la Facultad de Ciencias de la Comunicación de la Universidad Rey Juan Carlos (Madrid). España.

Correo: carlos.oliva.maranon@urjc.es
} 


\title{
SPORTS LANGUAGE AND SOCIAL COMMUNICATION: CONTEMPORARY PROTOTYPE OF MASSES
}

\begin{abstract}
The sport is conceived as an authentic Copernican revolution, interpreted as theory of the communication and, therefore, it is a part of the structure itself of the contemporary society. The interest by the sports language is based on the paradigm of which language and sport constitute two forms of intelligence and freedom. Today, the challenge of the New Technologies, the globalization of the sport and his great linguistic creativity that they form the need to control and to protect the language. The objective of this research is to know which are the principal linguistic mistakes of the sports information in the digital press. These deviations of the rules commit an outrage against the idiomatic correctness of the Spanish Language. On the one hand, the research reveales that the sports press includes terms of violent language, metaphors, gallicisms, anglicisms, omission of the article and incorrect use of the prepositions. On the other hand, in the sports language states the terminology of different areas as the religion, the health, the epic or the music. The reasons of these lexical mistakes are diverse: unconcern for the good use of the language and limitation of space in the news. All this, it concerns the area of the Humanities and, consequently, to the society.
\end{abstract}

KEY WORDS: Sports language - Sports Press - Spanish language - Foreign terms New Technologies

\section{INTRODUCCIÓN}

La palabra "deporte" proviene del latín deportare y no del vocablo inglés sport. La primera referencia a esta palabra se encuentra en la lengua provenzal y, de ella, deriva a otras lenguas romances como el francés o el castellano. Etimológicamente, esta palabra se utilizó para referirse a regocijo, diversión, recreo, etc. Por primera vez aparece atestiguada con este significado en un poema de Guillermo de Poitiers, IX duque de Aquitania y primer poeta de nombre conocido en las literaturas románicas. Posteriormente, en el libro de Los Milagros de Nuestra Señora de Gonzalo de Berceo, se referencia con el significado de diversión en forma de ejercicios físicos.

En la actualidad, el lenguaje periodístico-deportivo se caracteriza por ser una variante del uso común de la lengua. Parece ser una creencia cada vez más extendida entre gran parte de los lingüistas que el uso que hacen de nuestra lengua los medios de comunicación suele ser descuidado, e incluso, en determinadas ocasiones, incorrecto. Esta es una afirmación que se puede corroborar con facilidad. Algunos de esos errores tienen un clara "justificación": las prisas finales al cierre de la edición provocan que el proceso de escritura tenga que aligerarse, de manera que, precisamente, para evitar errores de tipo gramatical, impropiedades de estilo o sinsentidos semánticos, se acude al uso de modelos fijos de oraciones o de esquemas sintácticos efectivos que pueden 
utilizarse de forma recurrente para narrar un mismo tipo de noticia, a pesar de que esto constituya, muchas veces, un evidente obstáculo en la fluidez del estilo del texto. En otros casos, la limitación de espacio favorece un tipo de escritura compacto en el que, para evitar la repetición de distintos términos, la información se va acumulando mediante oraciones de relativo que pueden llegar a sobrecargar el texto (Forment, 1999, p. 185). La despreocupación por el buen uso de la lengua es un hecho patente en la sociedad española actual. La inflación de teoría lingüística en las aulas por un lado, y ese fenómeno que se ha hecho llamar "pasotismo", por otro, han afectado como no podía ser menos, al mundo de las Humanidades y, por ende, al del lenguaje.

El periodismo del primer tercio del siglo XX prestaba especial atención a la vida moderna y a sus cambios y observaba la vida internacional. Una de sus manifestaciones era el deporte, al que hubo que desarrollar entre numerosas generaciones de periodistas como un lenguaje de difusión social. En un primer momento, destacó la labor pionera de los diarios El Debate y La Veu de Catalunya.

No obstante, el lenguaje deportivo está cargado de fórmulas que se repiten con regularidad para las que los lingüistas emplean diversas denominaciones: metáforas, locuciones, giros, modismos, perífrasis léxicas, expresiones idiomáticas, etc. La importancia del deporte en los medios de comunicación es indiscutible; de tal manera que, el deporte, y no sólo el fútbol, forma parte de nuestras vidas. También, los políticos saben de su importancia, lo mismo que el empresario, el publicista, el médico, o el diseñador de moda, recurriendo a términos deportivos en sus expresiones coloquiales. Por lo cual, el deporte se concibe como una auténtica revolución copernicana, interpretado como teoría de la comunicación y, por tanto, es parte de la estructura misma de la sociedad actual.

\section{LOS MEDIOS DE COMUNICACIÓN Y EL DEPORTE}

La influencia que el deporte ejerce en los medios de comunicación se manifiesta principalmente en su programación y en su economía. El deporte, como consecuencia de su dimensión global, ha sido uno de los principales protagonistas de los procesos de implantación de las metodologías en la comunicación contemporánea (uso de los satélites, del vídeo, de las cámaras especiales). La consolidación de algunas cadenas de televisión y su popularidad se ha debido en parte a su protagonismo en la cobertura de grandes acontecimientos deportivos.

Con respecto a la definición del término deporte, se afirma:

"El deporte constituye una fuente inagotable de noticias para los medios, de gran interés popular, de alguna manera compensatorias o alternativas de las informaciones de actualidad siempre más conflictivas". (Moragas, 1994, p. 58).

Así, el deporte, primero en el caso de la radio y seguidamente en el de la televisión, ha sido una fuente inagotable de programas, de transmisiones en directo o en 
diferido que representa una parte cada día más importante del total de la emisión televisiva moderna. Las condiciones de la nueva ecología de la comunicación (necesidad de rapidez en la percepción de los mensajes publicitarios, impacto en los públicos -targets- con alta capacidad adquisitiva, calidad de imagen, competitividad entre cadenas, alternancia en el interés por lo local y lo global, etc.) reforzará aún más en el futuro esta aportación del deporte a las economías y a la programación de los medios de comunicación.

Los cambios empezaron a ser radicales cuando los estadios se transforman en platós de televisión. En este momento se inicia el proceso de adaptación de los calendarios deportivos nacionales e internacionales a las exigencias del calendario de los medios de comunicación, especialmente, de la televisión. Se busca la periodización y se evita el solapamiento entre acontecimientos. Se internacionaliza el calendario, los deportes occidentales se popularizan en los países orientales y la llegada de la televisión cambia en muchos países el índice de popularidad de los deportes. Poco a poco se produce la adaptación de los horarios del deporte a las exigencias o prioridades horarias de los medios de comunicación, especialmente, en el caso del prime time (horario estelar).

En este sentido, el profesor Antonio Alcoba afirma: "Ninguna otra actividad genera un mayor volumen informativo que el deporte" (Alcoba, 2005, p. 10). Además, con el objetivo de conectar mejor con la audiencia, de captar su atención y fidelizar su consumo, una buena parte del periodismo deportivo en Lengua Española ha optado decididamente por aproximarse a las formas más coloquiales del idioma y adoptar un estilo de narración que no sólo informe, sino que, además, sirva de entretenimiento. A este respecto, el Libro de Estilo de Canal Sur Televisión deja claro cuáles son los parámetros entre los que se ha de mover un periodista deportivo:

"El espectador espera tres cosas de una información deportiva: rememorar lo sucedido, datos novedosos y anécdotas y diversión y distracción, sobre todo, si el resultado es favorable. La obligación del periodista es cubrir estas expectativas y mediar entre el público y el deporte sin renunciar al rigor y la seriedad, pero sin olvidar la pasión y la expectación que disciplinas como el fútbol generan". (Canal Sur, 2004, p. 106 y ss.)

Este interés por el mundo deportivo lo incrementan la televisión de alta definición y los procesos de digitalización y multimedia, los cuales determinan nuevas formas de ver el deporte; aumentando sin duda su espectacularidad y, consecuentemente, su interés entre los telespectadores.

\section{EL LENGUAJE DEPORTIVO}

El desarrollo extraordinario de la información deportiva a ambos lados del Atlántico ha propiciado la creación de un lenguaje sectorial, con multitud de términos y expresiones nuevas, que ha evolucionado con personalidad propia en los últimos años hasta convertirse en terreno de avanzadilla de la lengua española. Efectivamente, los medios de comunicación deportivos contribuyen a diario a ensanchar el idioma 
creando usos lingüísticos, vocablos y acepciones nuevas que años más tarde, tras generalizarse y pasar a formar parte del acervo léxico común, acaban incorporándose al diccionario.

No obstante, también es considerado como una fuente permanente de problemas idiomáticos y se sitúa en el punto de mira habitual de filólogos y expertos en el uso del lenguaje por el abuso que comete de palabras extranjeras, de formas coloquiales que en algunos casos llegan a ser incluso vulgares-, así como por la pobreza del léxico empleado y la abundancia de errores gramaticales; rasgos que en algunos casos le han llevado a adoptar determinados usos que se apartan demasiado de la norma culta (Rojas, 2010, p. 5).

La escritura deportiva desempeña un nuevo papel social según los estudios del Centro de Estudios Olímpicos de Barcelona. El deporte, convertido en ritual, constituye con su propio lenguaje una realidad difundida masivamente a la sociedad por complejas relaciones tecnológicas, industriales y culturales. Se caracteriza por un conjunto de retos:

$\varnothing \quad$ Un primer reto es la relación con la informática emergente, con sus consecuencias de convergencia multimedia, mundialización del deporte y sus repercusiones culturales o la nueva relación de la experiencia del deporte con el espectador.

$\varnothing \quad$ Un segundo reto está formado por la publicidad y la comercialización, con el uso del idioma como escaparate de empresas de comunicación para la búsqueda de audiencias masivas, una compleja relación que produce mecanismos como:

$\S \quad$ Interrupción de la rutina.

$\S \quad$ La presentación del hecho en tiempo real.

$\S \quad$ La planificación minuciosa.

$\S$ La presentación reverente y ceremoniosa como hechos históricos.

$\S \quad$ La provocación de pasiones.

$\S \quad$ La celebración social del acontecimiento.

$\S \quad$ La promoción de la integración social.

$\S \quad$ La renovación de la lealtad hacia la sociedad.

$\varnothing \quad$ El tercer reto guarda relación con la creación de redes, servicios de comunicación y patrocinio.

$\varnothing \quad Y$, finalmente, están los retos de la política deportiva relacionados con el servicio universal, la financiación del deporte y las nuevas experiencias en comunicaciones y mensajes para el público.

La combinación de todos estos factores da lugar a nuevos tipos de relato informativo, sobre todo, en el ámbito audiovisual. El profesor José Polo, en su obra Lenguaje y 
Deporte, precisa esta caracterización y define el lenguaje deportivo como "un uso de la lengua común al que se han añadido una serie de rasgos específicos caracterizadores" (Polo, 1992, p. 40). Por su parte, el profesor Fernando Vilches afirma: "Mal está que algunos hinchas sean violentos, aunque poco podemos hacer la gente normal contra ello. Pero, sí se puede luchar contra la violencia en el lenguaje deportivo de los periódicos" (Vilches, 2004, p. 363).

De esta forma, la prensa deportiva, con el objetivo de entretener tanto o más que informar y de copar cuanto más mercado mejor dada la creciente competencia publicitaria, ha evolucionado adoptando algunos rasgos típicos de la prensa más populista o sensacionalista, a partir de un diseño llamativo que ha ido incorporando color, fotografía y todas las variantes tipográficas posibles.

Esta apuesta formal repercute igualmente en el tratamiento de los contenidos, alterando criterios objetivos de selección y valoración de la información, dentro y fuera del texto; dando cabida y relevancia a aspectos que a simple vista resultarían anecdóticos o secundarios en cualquier otro medio; y descuidando los controles de calidad a la hora de utilizar el lenguaje (Paniagua, 2003).

Por ello, el profesor Jesús Castañón hace algunas consideraciones:

"La consolidación de la lengua periodística como lengua de prestigio ha sido frecuentemente puesta en entredicho, puesto que se ha considerado a los medios de comunicación como agentes de deterioro del idioma. En este marco general, el periodismo deportivo ha sido acusado de empobrecer el vocabulario, de usar irresponsablemente los distintos niveles de la lengua y de favorecer una información dirigida al goce en vez de al desarrollo de la inteligencia en la lectura" (Castañón, 1993, p. 9).

También, Castañón nos cita:

"Idioma y deporte son dos manifestaciones de la inteligencia y la libertad. No frenan la exhuberancia, la orientan dirigen y dominan. En ellos, el esfuerzo equivale a progreso. La libre combinación de formas a creatividad. La originalidad, la emoción, y la competición, a una fiesta capaz de concentrar el pensamiento colectivo de los pueblos y de convertirse en una ocasión para producir arte". (Castañón, 1999).

Se podrían rastrear muestras de las variaciones características del lenguaje sectorial deportivo en cualquier ámbito de la lengua (fonético-fonológico, morfosintáctico o léxico-semántico). Sin embargo, resulta evidente manifestar que esos usos peculiares son especialmente relevantes en el ámbito léxico. Algunos lingüistas consideran que no existen usos especiales ni sectoriales de la lengua, sino parcelas específicas de vocabulario y conjunto de términos característicos de diferentes áreas o actividades. Las peculiaridades del lenguaje del deporte serán más notables en el ámbito léxico- 
semántico que en cualquier otro.

\subsection{Características del lenguaje deportivo}

En el lenguaje deportivo están ya institucionalizadas formas abreviadas de antropónimos como Zizou (Zidane), Becks (Beckham), Dinho (Ronaldinho) Mou (Mouriño) y Pep (Guardiola). Como también lo están los apócopes de clubes, que incluso llegan a desplazar casi definitivamente de los titulares a las nóminas completas y reales de cada entidad. Así ocurre, por ejemplo, con la Juve (Juventus), el Barça (Barcelona), el Depor (Deportivo), el Poli (Polideportivo Ejido), el Estu (Estudiantes), el Fuenla (Fuenlabrada) o el Geta (Getafe).

Otras características de este lenguaje, en opinión de Fernando Lázaro Carreter, son las siguientes (Lázaro Carreter, 1994, p. 25):

$\varnothing \quad$ Abuso del epíteto que aporta color:

$\S \quad$ El césped del estadio es un verde tapiz.

$\S \quad$ Un gol es de bella factura.

$\S \quad$ La carrera ciclista adquirió un ritmo trepitante.

$\S \quad$ Hay jugadores que son extremos natos o goleadores natos.

$\varnothing \quad$ Presencia de metonimias (figura consistente en designar el todo por la parte), como bien se aprecia al emplear las palabras cuero para designar al balón; trencilla, al árbitro; meta, al portero, etc.

$\varnothing \quad$ La elusión que alude, o evoca, al lugar de origen: los de Pucela, los de la ciudad Olívica., para indicar a los jugadores del Valladolid y del Celta de Vigo, respectivamente.

$\varnothing \quad$ Por el camino de la ilusión se llega a la antonomasia, que siempre ha gozado de resultados bastantes estables en la cultura occidental. Fue un recurso utilizado por los juglares de la Edad Media: Ruy Díaz era el de Vivar.

$\varnothing \quad$ En el registro deportivo, por ejemplo, es una constante identificar al ciclista Federico Bahamontes como el Águila de Toledo.

$\varnothing \quad$ Luis Aragonés, como El Sabio de Hortaleza.

$\varnothing \quad$ Los pupilos de (+ el nombre del entrenador) para referirse a un equipo concreto.

$\varnothing \quad$ Las victorias alcanzadas son brindadas por los atletas actuales a las Vírgenes de distinta advocación, y la emoción aumenta con el énfasis y la desmesura que encierran las hipérboles como la del siguiente titular:

$\S \quad$ El Madrid alfombró con goles su camino hacia la Copa del Rey.

$\S \quad$ Otras veces, se resalta la decisión de ardor deportivo: Los chicos de Guardiola fueron un vendaval.

$\S \quad$ Se expresa la efectividad de la resolución: Cristiano apuntilló a los valencianos.

$\S \quad$ No se anduvo con chiquitas: el Málaga recibió un duro mazazo...

Por tanto, el lenguaje del deporte es un lenguaje pintoresco, exagerado, pomposo, 
casi extravagante en algunos casos, pero extremadamente rico al mismo tiempo. En este sentido, el profesor Josep María Casasús afirma:

"El periodismo deportivo, basado en los lenguajes verbal y visual, es hoy uno de los grandes vehículos de la ética entendida como compromiso responsable del individuo con la sociedad" (Casasús, 2006, p. 50).

La lengua de la prensa deportiva se caracteriza por la abundancia de términos sinónimos, riqueza que no manifiesta falta de precisión en la expresión, sino, por el contrario, variedad estilística y connotación a través de la multiplicidad denominadora. Así, por ejemplo:

$\varnothing \quad$ Balón, además de recibir esa denominación, también puede ser llamado la pelota, el esférico, la bola, el cuero, etc.

$\varnothing \quad$ El campo que, además, puede ser el estadio, el terreno de juego, el césped, la cancha.

El léxico del deporte por sus peculiaridades específicas presenta un importante número de préstamos lingüísticos, fundamentalmente procedentes del inglés, y que es excesivo según la opinión de algunos autores. En muchas ocasiones esos préstamos han convivido y han llegado a desplazar al correspondiente término autóctono al que los hablantes han considerado mucho menos expresivo y efectivo:

$\varnothing \quad$ (fútbol / balompié; básket / baloncesto, etc).

La mayoría de ellos han sido adaptados a las características fonéticas de nuestra lengua $y$, a partir de esa adaptación el sistema, han originado algunos términos derivados, utilizando para ello métodos y partículas especialmente españoles:

$\varnothing \quad$ "Goal" golear, goleada, goleador, golazo, hombre-gol.

$\varnothing \quad$ "Doping" dopar, dopaje, dopado, control antidoping / antidopaje.

Los términos utilizados en las crónicas deportivas no pueden ser clasificados casi nunca como vocablos neutros. Un ejemplo claro lo apreciamos en la adjetivación que suele ser abundante y también fuertemente marcada positiva o negativamente. En las crónicas deportivas son frecuentes elementos como los siguientes:

$\varnothing$ Tosco, espeso, obcecado, miserable, caótico, desconcertante, estéril, interminable, coja, humillante...

Frente a:

$\varnothing \quad$ Luminoso, fulgurante, lúcido, inteligente, genial, brillante, soberbio... La neutralidad tampoco caracteriza a los verbos que aparecen en la prensa deportiva. Basta pensar en las formas:

$\varnothing \quad$ Sucumbir, arrancar, (un punto), aplastar, sentenciar, embarrancar, 
fundir, rematar, apoderarse, conquistar...

La presencia, abusiva en algunos casos, de estas formas marcadas pretende conferir al texto en el que aparecen una mayor viveza, un mayor grado de expresividad. Su búsqueda provoca el ensalzamiento de cualquier elemento que pueda ser considerado llamativo. En este sentido, cabe destacar la utilización de términos pertenecientes a otros campos semánticos que se trasladan al vocabulario deportivo, aumentando así considerablemente su poder provocador. Algunos ejemplos destacados agrupados por campos semánticos son los siguientes:

\subsubsection{Vocabulario procedente del ámbito religioso}

$\varnothing \quad$ El cambio de un jugador puede ser una incorporación divina, una milagrosa reaparición.

$\emptyset \quad$ Un jugador que embiste con fe.

$\varnothing \quad$ La resurrección de un equipo en una determinada competición.

$\varnothing \quad$ La visita del Zaragoza al Camp Nou dejó legado la comunión entre grada y cancha y un punto de inflexión en un Club azotado por las fraticidas.

$\varnothing \quad$ Renacen las virtudes teologales en un colectivo que renegaba de su carnet ante el altar.

\subsubsection{Vocabulario del campo semántico de la salud}

$\varnothing \quad$ Enfrentarse a un rival al borde de la defunción.

$\varnothing \quad$ Un equipo puede ser un enfermo que amenaza con entrar en fase terminal.

$\varnothing \quad$ Un equipo pretende seguir vivo en una determinada competición.

$\varnothing \quad$ Un partido adquiere un tono agónico.

$\varnothing \quad$ Un jugador renacido.

$\varnothing \quad$ Salir tocado (un equipo) de un encuentro.

\subsubsection{Vocabulario relacionado con situaciones bélicas y procedentes de la épica}

$\varnothing \quad$ Un disparo, lanzarse al ataque, poner cerco al área contraria, amurallar el área, ganar terreno al rival, colocar una tupida defensa, recobrar el aliento tras la batalla, un partido ganado a base de tesón y coraje, atrincherarse en la zona de los medios, el feudo de un equipo, el héroe del conjunto, vencer al enemigo, infiltrarse en campo contrario.

$\varnothing \quad$ Términos como fortaleza, pugna, contienda, verdugo, gesta, duelo, que habían dejado de utilizarse en la lengua común bien porque habrían sido consideradas por parte de los hablantes como formas arcaicas han recuperado fuerza y uso a través de la utilización que de ellos se hace en el leguaje deportivo. 


\subsubsection{Vocabulario procedente del ámbito musical}

$\varnothing \quad$ Dar un recital de buen juego, dar el do de pecho, llevar la batuta, sintonía de un partido, hacer una dirección brillante del encuentro, desafinar, llevar el compás...

La diversidad que muestra el lenguaje del deporte se combina con el uso de una fraseología que no difiere excesivamente de la lengua común, sino que es ella misma, aplicada a diferentes situaciones en virtud de símiles. Algunos ejemplos son:

$\S \quad$ Tener el triunfo en las botas, por (en las manos).

$\S \quad$ Estar con el agua al cuello (un equipo, una entidad).

$\S \quad$ Hacer aguas (la defensa).

$\S \quad$ El árbitro casero barre para casa.

$\S \quad$ Dejarse la piel (en el campo).

$\S \quad$ Emplearse a fondo.

$\S \quad$ Complicarse la vida (a pesar de que el partido es fácil). restantes).

$\S \quad$ Poner tierra de por medio (entre el primer clasificado y los seguidores

$\S \quad$ Llover sobre mojado.

\section{PRÉSTAMOS DE VOCABULARIO ENTRE DISTINTOS DEPORTES}

De entre todos los espectáculos deportivos que se organizan habitualmente en Europa, ninguno alcanza las dimensiones del fútbol; ninguno engendra tantas pasiones, tanta crispación, tanto arrebato, tanto fervor, ninguno consigue congregar en los campos o frente al televisor a tantos miles de aficionados.

Como espectáculo de masas, el fútbol ha superado al cine -que parecía destinado a erigirse en el entretenimiento por excelencia del siglo XX- y ha ido creando una espesa red de intereses comerciales que lo han convertido, en muchos casos, en una empresa económica de primer orden. Los medios de comunicación les conceden un espacio que sobrepasa al de cualquier otra sección informativa (en los Telediarios hay un periodista especializado en deportes que comenta la sección deportiva, cuya duración excede de las otras secciones informativas).

El fútbol ha ido incorporando usos léxicos ya consolidados en otros deportes: diana.

$\varnothing \quad$ Tener un pinchazo, noquear, quedar fuera de combate, derby, hacer

También, procedentes del campo bélico, lo que se explica por el carácter de enfrentamiento que posee cualquier encuentro:

$\varnothing \quad$ Fusilar (un gol), rematar, disparar, cañonazo, derrotar, capitanear.

El compuesto balompié, traducción de la voz inglesa, representa la respuesta autóctona al primitivo barbarismo fútbol que no ha perdido continuidad en la 
información periodística. Al comenzar la temporada futbolística en Inglaterra, los grandes terrenos deportivos vuelven a verse invadidos del público devoto del balompié.

En otras ocasiones, se emplean términos de carácter peyorativo o con connotaciones violentas. Veamos el ejemplo de la voz 'hooligan', una de las palabras más utilizadas hoy día cada vez que se tratan noticias referidas al fútbol británico. Al contrario de lo que muchas veces dan a entender los medios de comunicación, este vocablo no es sinónimo de 'hincha', sino de 'gamberro', 'ultra' o 'seguidor violento'. Por tanto, no es adecuado generalizar y decir que todos los aficionados de un determinado equipo británico son 'hooligans', sino -puestos a utilizar la palabra inglesa exacta- 'supporters' (Rojas, 2005, p. 63).

Por su parte, Manuel Vázquez Montalbán halla en el deporte "rey" el terreno abonado para el desarrollo de la violencia por diversos motivos:

"El fútbol ha sido el derecho a la épica, ejercido a tontas y a locas por el pueblo. Ha sido, y es, un instrumento de desviación de la agresividad colectiva hacia un cauce no político. Pero también ha servido, juzgado desde otra perspectiva, como válvula de escape de las frustraciones del hombre de la calle y, por lo tanto, ha cumplido un papel higiénico sobre la prenormal conciencia social del país". (Vázquez, 2006, p. 75).

La riqueza que caracteriza el estilo y el léxico del lenguaje del deporte puede ocasionarse por distintos motivos: en la mayoría de los casos, las hipérboles y florituras del estilo de la lengua del deporte son elegidas de manera deliberada, es decir, el periodista emplea voluntariamente un registro determinado para redactar sus textos con la clara voluntad de atrapar al lector, oyente o espectador. Otras veces, por el contrario, estos elementos están motivados por la improvisación necesaria en determinadas situaciones, para intentar ganar tiempo... en definitiva, porque en algunos contextos -orales fundamentalmente- podemos afirmar que el lenguaje del deporte no se diferencia del español hablado más que en el uso de algunas voces o partículas características del sector o jerga analizado.

\section{LA POBREZA LÉXICA DIFUNDIDA A TRAVÉS DE LOS MEDIOS}

El lenguaje incorrecto es hoy patente, especialmente, en el ámbito juvenil. Las conversaciones de los jóvenes suelen estar salpicadas de palabras cliché y tópicos (cosa, rollo, chisme, tío, colega, mogollón, chorrada), de adjetivos semánticos descoloridos (divino, precioso, fantástico...), de locuciones o frases hechas (lo típico, dar corte, o sea), de "tics" interjectivos o fáticos, de muletillas, de repeticiones innecesarias, etc.

"A la pobreza léxica se une como señal de alarma el cúmulo de impropiedades en los significados de las palabras, que con tanta 
frecuencia se desprenden de comentarios en radio, televisión y medios de comunicación en general" (Gómez Torrego, 1995, p. 7).

Especialmente, estas variantes se reflejan en los comentarios deportivos de los que los jóvenes son grandes consumidores. Sobre esta misma cuestión, Álex Grijelmo señala que el periodista debe mostrar un lenguaje común, en ningún caso vulgar ni pobre, que tienda hacia la precisión y resulte eficaz en la elección de los significados concretos y adecuados:

\begin{abstract}
"Sobre todo en el lenguaje deportivo, los periodistas acuden insistentes a expresiones de la calle que dicen muy poco de su vocabulario personal (...) El informador no puede permitir que le contagien las palabras que usan sus amigos o su entorno ("le metía 16 segundos" forma parte de un lenguaje vulgar o de jerga que el redactor no puede asumir)". (Grijelmo, 2000, p. 342).
\end{abstract}

La definición del género de la crónica deportiva se podría considerar como la narración de un hecho de forma continuada, si nos atenemos a su origen etimológico. Según Martín Vivaldi,
"la palabra crónica tiene un origen histórico-literario antes de su adaptación al periodismo tal y como hoy la conocemos. Su origen se remonta a los relatos de la época clásica, en la que los griegos y los romanos contaban hechos bélicos narrados por sus protagonistas o testigos de ellos, con un orden temporal" (Martín Vivaldi, 1998, p. 123).

Por su propia etimología, estamos ante relatos en los que se observa una sucesión temporal de acontecimientos, con un hilo conductor. Relatos cronológicos referidos a lo sucedido entre un período delimitado (hora, día, semana...). Así, Bernal Rodríguez la entiende como "una información de hechos noticiosos, ocurridos en un período de tiempo, por un cronista que los ha vivido como testigo, investigador, e incluso, como protagonista y que al mismo tiempo que los narra, los analiza e interpreta mediante una explicación personal" (Bernal, 1997, p. 27).

Frente a otros géneros periodísticos, la crónica en el deporte permite cierta libertad a la hora de escribir o hablar de eventos deportivos, al no precisar ceñirse de manera estricta a la actividad deportiva.

Según Lázaro Carreter, "los dislates se acumulan en transmisiones y crónicas" (Lázaro Carreter, 1994, pp. 19-35):

$\varnothing \quad$ Desde el "reiniciar", con que han sustituido a reanudar (porque indicaría que se vuelve al principio).

$\varnothing \quad$ "Este área", en lugar de esta área, y llegando a ese verdadero "genocidio" de los verbos que significan: "Empezar" y "acabar", sacrificados todos a los omnipresentes iniciar y finalizar. 
$\varnothing \quad$ Las faltas no se "señalan", sino que se señalizan.

$\varnothing \quad$ Puede escribirse impunemente que la Liga se ha convertido en un "duelo a tres", sin reparar que el duelo es cosa de dos.

En España, salvo en los tiempos prehistóricos de sus comienzos cuando los castizos decían "footbal", siempre se ha dicho fútbol. Jamás se popularizó la castellanización balompié, salvo excepción que confirma la regla, en el nombre oficial del muy popular Real Betis Balompié. El fútbol no sólo ha incorporado el anglicismo que le da nombre al lenguaje cotidiano, sino otros muchos términos como penalti, al que el tiempo y la Academia han hecho caer la -y- griega modificándola por la -i- latina.

El tiempo también ha dejado en la cuneta muchas palabras, expresiones y términos deportivos y ha aportado otras nuevas. En baloncesto se utilizaba la palabra "cámino" en los años setenta. Fue en aquellos años cuando el baloncesto rompió la barrera de lo minoritario, donde se hallaba inmerso y pasó a ser conocido de forma masiva. Hoy apenas se utiliza el vocablo "cámino" y se habla de "pasos". A cambio de la desaparición de "cámino", se apodera del lenguaje el anglicismo "basket" como sinónimo de baloncesto. Basket, en castellano, es cesta, también papelera, y cierto que la cesta es el elemento básico de este deporte. Tenemos de todo.... La calle impone sus normas y a la gente joven le gusta calzar zapatillas de basket o llevar unas baskets. En Iberoamérica, al baloncesto se le conoce como básquet, con "qu" no con "ka" y a los practicantes se les conoce como basquetbolistas, vocablo que admite la Academia.

\section{LOS TECNICISMOS EN EL LENGUAJE DEPORTIVO}

En opinión del profesor Martínez Albertos,

"el lenguaje periodístico es un lenguaje mixto en el que se dan códigos concurrentes que hacen que los diferentes lenguajes se condicionen (Martínez Albertos, 2001, p. 203).

Por ello, al mismo tiempo que el lenguaje deportivo deja huella en otros lenguajes, estos otros también lo influencian, pues la intertextualidad o uso trasladado de un léxico específico a otro es un rasgo constante entre las lenguas especializadas que conforman la lengua de los medios de comunicación (Guerrero Salazar, 2002, pp. 368374).

En el lenguaje deportivo, la radio fue el primer medio que divulgó el uso de la metáfora. La necesidad de visualizar el juego por medio de la palabra creó la imagen. La prensa, después, se ha aprovechado de los logros radiofónicos. El lenguaje deportivo se sirve, sobre todo, de las metáforas usuales o lexicalizadas, es decir, desvío de primer orden, habituales y comunes, pero no muertas. En ellas predomina lo afectivo, más que lo formal, pues acostumbran a ser muy sencillas estructuralmente: simples sustituciones o metáforas copulativas (Hernández, 2003, p. 68). 
A veces la variedad léxica convive con textos de carácter "pseudoliterario", en los que predominan la connotación, el uso ornamental del adjetivo, las figuras retóricas, los juegos de palabras, etc. Relacionada con los juegos de palabras está la modificación del discurso repetido. Castañón Rodríguez ha aludido a ello como una característica de la escritura deportiva. De hecho, es usual que en las crónicas y en los titulares se modifiquen unidades de lengua coloquial como giros, refranes o palabras extranjeras (Castañón, 1999, p. 204).

En el idioma español, el número de anglicismos ha aumentado considerablemente, también, en el francés. En cuanto a vocablos franceses, en deportes, la palabra más extendida ha sido "elite", "selecto", en gran parte acentuando la "e" primera y pronunciando la última:

$\varnothing \quad$ "Si no puedes marcar gol con ciencia, lo haces hasta con el juanete; eso hacen los equipos de elite".

$\varnothing \quad$ El "grand-prix", pronunciado "gran-pri" o "gran-pris", es el gran premio que se otorga a un tenista, motorista, ciclista, etc.

$\varnothing \quad$ La palabra "rol" que en francés es "rôle", con " $\mathrm{e}$ " final equivale a "un papel" desempeñado, con lo que queda claro que es una pedantería o una ignorancia.

$\varnothing \quad$ Esto también se manifiesta en el caso de la palabra "honesto" que en español tiene varias acepciones; el francés lo emplea en casi todos los casos "honnête" y el español imita con "honesto", lo que hace desaparecer el concepto de "púdico".

$\varnothing \quad$ La palabra débâcle, "debacle", en el sentido de "derrota", no se oye mucho en la calle, pero sí aparece en prensa, radio y televisión.

Leonardo Gómez Torrego llama impropiedades léxicas "al empleo de ciertos vocablos con significados o matices significativos que no les corresponden" (Gómez Torrego, 1995 , p. 15). Impropiedades léxicas que se cuelan con mucha frecuencia en los medios de comunicación y, de forma especial, en los locutores deportivos. El autor señala las siguientes causas: la pedantería, la ignorancia, las etimologías populares, las paronimias y las influencias foráneas.

\section{EXPRESIONES EN LA PRENSA DIGITAL DEPORTIVA}

\subsection{Lenguaje violento}

$\S \quad$ Una venganza para empezar

(La Razón 2-1-2010)

$\S \quad$ Los cañoneros se dan otro festín

(Marca 6-4-2010)

$\S \quad$ Messi revoluciona al Barça

(Marca 7-11-2010)

\subsection{Metáforas}

$\S \quad$ El Atlético hace aguas 
(La Razón 22-3-2010)

$\S \quad$ El tanto espabiló a los ovetenses

(Marca 5-4-2010)

$\S \quad$ Más dinamita que buen juego

(Marca 21-3-2010) acabó...

$\S \quad$ A partir de ese momento, el jugador murciano apretó los dientes y

(Marca 13-1-2010)

\subsection{Galicismos}

$\S \quad$ La marca japonesa Honda logra obtener un comprador de cara a (PARA) competir en el Mundial de Fórmula Uno del año que viene.

(El País 8-1-2010).

\subsection{Gerundio}

$\S \quad$ Se había sacado el casco y se refugió en la sombra, disponiendo de comida y alimentos encontrados cerca de él.

(ADN 8-1-2009)

\subsection{Sintaxis}

$\S \quad$ El Málaga escapa del descenso, tres meses, después ante el Getafe. (La Razón 5-4-2010). (Redacción errónea).

$\S \quad$... Y el pívot serbio haciendo blanco con cualquier griego que andara (ANDUVIERA) cerca, el corro se deshizo.

(La Razón 21-8-2010)

$\S \quad$... Es uno de los delanteros más efectivos de la Liga española, si no (SINO) que es el auténtico revulsivo que necesitaba el Real Madrid, que ha ganado, tras caer en Barcelona, sus últimos tres partidos ligueros.

(La Razón 21-12-2009)

$\S \quad$ Suya fue la jugada del primer gol, obra de Di María, y fue él, quién sino, (SI NO) el que asumió la responsabilidad de chutar el penalti en el último minuto.

(La Razón 10-2-2011)

$\S \quad$ Ha rendido en la selección siempre y cuando no ha jugado, nunca se ha quejado.

(La Razón 15-3-2007) (Redacción errónea)

\subsection{Anglicismos}

$\S \quad$ No se lo toman seriamente cuando sus coaches les dicen que los tiren al final de los entrenamientos.

(El País 14-1-2009) 


\subsection{Omisión del artículo}

Una desviación léxica muy frecuente en el lenguaje deportivo es la omisión del artículo, en frases como:

$\S \quad$ El balón se pierde por _ línea de fondo. (LA)

$\S \quad$ Termina _ encuentro en San Mamés. (EL)

$\S \quad$ El portero no se ha movido bajo _ palos. (LOS)

$\S \quad$ Maxi Rodríguez se sinceró ayer en _ rueda de prensa (LA)

$\S \quad$ El portugués se convirtió en _ portavoz del vestuario colchonero (EL)

$\S \quad$ Suyo fue el gol de la victoria, el segundo que marca en _ Liga. (LA)

$\S \quad$ A veces se producen esqueléticas elipsis: Va a sacar. Lo hace. Pierna izquierda...

\subsection{Uso incorrecto de las preposiciones}

No menos extravagante resulta ver sustituidas las preposiciones por SOBRE, que desempeña las funciones de todas las demás:

$\S \quad$ Puyol hace falta sobre (CONTRA) Casillas.

$\S \quad$ El árbitro pita falta sobre (ANTE) Villa.

$\S \quad$ Ya están los equipos sobre (EN) el terreno de juego.

\section{CONCLUSIONES}

El deporte se ha convertido en una fuente de cultura popular. Con el término "deporte" se designa un tipo de actividad con unas características determinadas, aunque la propia definición es origen de múltiples controversias entre distintos autores. En el marco de la filosofía del lenguaje, desde Herder y Humboldt hasta la moderna teoría de los campos lingüísticos, se sabe muy bien que la forma del entendimiento guarda relación con la forma del lenguaje, el cual, influye notablemente sobre el pensamiento. Por consiguiente, no es casual que el deporte tenga su origen en la Grecia Clásica. Si tomamos como hipótesis la idea de que la consideración griega surge de su lenguaje filosófico y científico ligado a la comunidad, no al individuo, el deporte nace de la expresión de este concepto social, que ha sido el núcleo intelectual y se asienta en la necesidad de comunicación.

Respecto al léxico deportivo, los Medios de Comunicación Social (prensa, radio y televisión), en los espacios de información especializada, están "plagados" de incorrecciones lingüísticas. El lenguaje del deporte está formado por fórmulas lingüísticas fijas, cuyo objetivo es llamar la atención del lector. Algunos autores han caracterizado al lenguaje deportivo como "una variante del uso común de la lengua", por lo que, este lenguaje presenta términos prestados de ámbitos diversos: la religión, la salud, la épica o la música; así como tecnicismos importados del inglés y del francés. Es, por tanto, una lengua técnica, que relata hechos $y$, también, trata de presentar vivamente ante el espectador la actuación de los jugadores reflejando en ella la emoción de la contienda. 
Así, al margen de las incorrecciones léxicas, el deporte se manifiesta como un tejido de ideas para representar el propósito a través de la corporeidad que, sin él, sería un fenómeno privado. Sin duda, el deporte ha llegado tan lejos al convertirse en una vivencia cultural, un tema de conversación con gran repercusión social y un recurso para la redacción de otras áreas informativas. En definitiva, pocos contactos, confrontaciones $\mathrm{o}$ intercambios internacionales tienen tanta resonancia entre las masas como los acontecimientos deportivos.

\section{REFERENCIAS}

Alcoba, A. (2005): Periodismo deportivo. Síntesis. Madrid.

Bernal, M. (1997): La crónica periodística: tres aproximaciones a su estudio. Padilla. Sevilla.

Canal Sur Televisión (2004): Libro de Estilo de Canal Sur televisión y Canal 2 Andalucía. RTVA. Sevilla.

Casasús, J.M. (2006): "La ética en el lenguaje deportivo verbal y visual" en El idioma español en el periodismo deportivo. Universidad Internacional Menéndez Pelayo. Santander.

Castañón, J. (1993): El lenguaje periodístico del fútbol. Universidad de Valladolid. Valladolid.

Forment, M. (1999): "Apuntes sobre la norma lingüística y el estilo del lenguaje del deporte y de la prensa del corazón" en Garrido, J. (1999): La Lengua en los medios de comunicación: actas del Congreso Internacional celebrado en la Universidad Complutense de Madrid en 1996 (pp. 183-193). Universidad Complutense, Servicio de Publicaciones. Madrid.

Gómez, L. (1995): "Impropiedades léxicas" en El léxico en el español actual: uso y norma (pp. 15-164). Arco-Libros. Madrid.

Grijelmo, A. (2000): La seducción de las palabras. Taurus. Madrid.

Guerrero, S. (2002): "El lenguaje deportivo, entre coloquial y literario" en Isla de Arriarán. Revista Cultural y Científica, Vol. XIX, 1. Pág. 365-382.

Hernández, N. (2003): El lenguaje de las crónicas deportivas. Cátedra. Madrid.

Lázaro, F. (1994): "El español en el lenguaje deportivo" en González, C. y Gómez, A.: El idioma español en el deporte (pp. 19-35). Fundación EFE. Madrid.

Martín, G. (1998): Géneros periodísticos, reportaje, crónica, artículo: análisis 
diferencial. Paraninfo. Madrid.

Martínez, J. L. (2001): Curso general de Redacción Periodística. Paraninfo. Madrid.

Moragas, M. (1994): "Deporte y Medios de Comunicación: sinergias crecientes" en Revista Telos: Cuadernos de Comunicación, Tecnología y Sociedad, no 38 de junio de 1994. pág. 58-62.

Paniagua, P. (2003): Información deportiva. Especialización, géneros y entorno digital. Fragua. Madrid.

Polo, J. (1992): Lenguaje y Deporte. Gobierno de La Rioja, Consejería de Educación, Cultura y Deportes. Logroño.

Rojas, J. L. (2005): La información y el deporte. Libro de estilo para la prensa deportiva andaluza. Aconcagua. Sevilla.

(2010): "La responsabilidad social del informador en el uso del lenguaje ante la proliferación de hechos violentos en el mundo del deporte" en Revista Vivat Academia, no 111. Julio-Agosto 2010. Disponible en:

www.ucm.es/info/vivataca/numeros/n111/DATOSS.htm

(Consultado el 20/02/2012).

Vázquez, M. (2006): Fútbol, una religión en busca de un dios. De Bolsillo. Barcelona.

Vilches, F. (2004): El menosprecio de la lengua. El español en la prensa. Dykinson. Madrid.

www.larazon.es (Consultado el 22/08/2010)

www.elpais.es (Consultado el 12/01/2011)

www.adn.es (Consultado el 14/01/2011)

www.marca.es (Consultado el 22/04/2010)

www.basketconfidencial.com/articulo/esqueletos+de+palabras_2021.html (Consultado el $18 / 02 / 2012$ )

www.idiomaydeporte.com (Consultado el 20/02/2012)

\section{Carlos Oliva Marañón}

Doctor en Ciencias de la Comunicación. Profesor de la Facultad de Ciencias de la Comunicación de la Universidad Rey Juan Carlos en las áreas de Periodismo, Comunicación Audiovisual y Magisterio. Autor, entre otras publicaciones, de las siguientes: Interacción del público en un entorno virtual. Comunicación interna y 
externa en el Museo Reina Sofía; Calidad, visibilidad e interactividad: paradigmas de las Universidades Virtuales en España; Sociólogos: competencias, formación universitaria y sinergias cognitivas en el Espacio Europeo de Educación Superior (EEES); Fundamentos lingüísticos del lenguaje periodístico y jurídico-administrativo; y La creación léxica mediante siglas: un recurso de condensación expresiva en la prensa digital. Ha trabajado en diferentes Organismos Oficiales como la Consejería de Educación de la Comunidad de Madrid, las Cortes Generales y el Instituto Cervantes. 